\title{
Seguimiento anual de la parasitosis gastrointestinal de venados cola blanca Odocoileus virginianus (Artiodactyla: Cervidae) en cautiverio en Yucatán, México
}

\author{
Rubén C. Montes Pérez, Roger I. Rodríguez Vivas, Juan Felipe de J. Torres Acosta y Lucy G. Ek Pech ${ }^{1}$ \\ 1 Facultad de Medicina Veterinaria y Zootecnia. Universidad Autónoma de Yucatán. Apartado Postal 4-116. CP. 97100. \\ Mérida, Yucatán, México. Fax (99) 423 205. Correo electrónico: mperez@tunku.uady.mx.
}

Recibido 4-XII-1997. Corregido 8-VI-1998. Aceptado 12-VI-1998.

\begin{abstract}
Gastrointestinal parasites, and egg and oocyst output in the faeces of captive white-tailed deer (Odocoileus virginianus yucatanensis) were recorded in Yucatan, Mexico. Feces were obtained from from January through December 1995 (ten samples every two weeks per place). Samples were processed by flotation and the McMaster techniques. Faecal cultures for $\mathrm{L}_{3}$ larvae were made by the Corticelli-Lai technique. Oocysts in faeces were cultured in $2 \%$ potasium dicromate. Seven genera were determined (Haemonchus spp., Cooperia spp, Isospora spp., Eimeria spp., Trichuris spp., Strongyloides spp. and Moniezia spp.) which represent five orders. The most frequent genera were Haemonchus, Isospora and Eimeria. The genus Isospora is reported for the first time in deer of this region, although it was not possible to explain the source of this parasite. The frequency and level of faecal egg and oocyst outputs were variable during the year and increased during the rainy season. There was a positive correlation between relative humidity, environmental temperature and rainfall with the coccidia and strongylida orders. In the central zone of Yucatan the meteorological conditions during the rainy season are favourable for the development of gastrointestinal parasitism which enable an increased risk of infection for deer.
\end{abstract}

Key words: Gastrointestinal parasites, Odocoileus, Strongylida, white-tailed deer, Coccidia, Helminthes.

La parasitosis gastrointestinal (PGI) en venados cola blanca (Odocoileus. virginianus), afecta a poblaciones libres y cautivas (Prestwood et al. 1973, Rogel y Luján 1982). Estudios realizados en venados cola blanca libres en reserva natural de los Estados Unidos de Norteamérica (Woolf y Harder 1979), indican que la mortalidad de venados por causa de PGI es baja, alrededor de $2.7 \%$, la cual representa el séptimo lugar de causas importantes de mortalidad en esta población. En México no se han registrado datos acerca de la mortalidad de venados por causa de PGI mantenidos en condiciones libres o en cautiverio; sin embargo, se menciona que puede representar un serio problema para animales confinados en zoológicos y criaderos (Roa 1986).

Los estudios registrados en México por Carrillo (1987), Domínguez et al. (1988), Rivera (1991), Olvera (1991) y Jaimes et al. (1993) indican la presencia de PGI de los órdenes Coccidia, Strongylida, Rahbditoidea, Enoplida y Anoplocephalidea. Los principales géneros de éstos son: Eimeria, Haemonchus, Oesophagostomum, Ostertagia, Trichostrongylus, Strongy- 
loides, Cooperia, Nematodirus, Chabertia, Capillaria, Toxocara, Moniezia, Trichuris, Bunostomum y Metastrongylus. Ninguna de las investigaciones realizadas en venado cola blanca en esta región describen las variaciones en la frecuencia de animales parasitados, ni las cargas parasitarias presentes en esos animales a lo largo de un ciclo anual; de tal manera que no se tiene algún dato que permita suponer los cambios parasitarios esperados para el siguiente ciclo. Si se contara con esta información, sería posible iniciar estrategias para el control de la parasitosis en venado cola blanca mantenidos en cautiverio. Con tal propósito se realizó este trabajo, con los siguientes objetivos: determinar los géneros de helmintos y coccidias que parasitan el tracto gastrointestinal de venados cola blanca mantenidos en cautiverio en el estado de Yucatán, México; medir las variaciones en las frecuencias de animales parasitados, asi como la cantidad de ooquistes y huevecillos excretados a lo largo de un año; relacionar la humedad relativa, temperatura ambiental y precipitación pluvial con las variaciones en las frecuencias de animales parasitados.

\section{MATERIALES Y METODOS}

Se colectaron muestras fecales frescas de venado cola blanca (Odocoileus virginianus yucatanensis) en tres cautiverios ubicados en la zona centro del estado de Yucatán, México. El clima de la región es tropical subhúmedo con lluvias en verano; clasificación climática de Köppen Awoig, modificada por García (1988). La temperatura ambiental máxima varía de 35 a $40^{\circ} \mathrm{C}$ y la temperatura ambiental promedio es de $27^{\circ} \mathrm{C}$. La humedad relativa varía de 65 a $90 \%$, con promedio de $80 \%$ y precipitación pluvial anual total de $935.5 \mathrm{~mm}$. Se presentan dos estaciones anuales: de lluvia (de junio a noviembre) y seca (de diciembre a mayo) (Anónimo 1970, García 1988).

Se muestrearon dos zoológicos y un criadero bajo un sistema de producción semi-intensivo. Estos confinamientos fueron denominados $\mathrm{C} 1$, $\mathrm{C} 2$ y $\mathrm{C} 3$ respectivamente. El $\mathrm{C} 1$ corresponde al
Parque Zoológico del Centenario; el cual está localizado en la Ciudad de Mérida, Yucatán, México. El C2 es el Zoológico de Cholul, ubicado en Cholul, municipio de Mérida, Yucatán, México. El C3 es un criadero perteneciente a la empresa Cementos Maya, ubicado en la zona industrial de la ciudad de Mérida, Yucatán, México. C1 disponía de diez venados, $\mathrm{C} 2$ diez y $\mathrm{C} 3$ diecisiete; entre los cuales había juveniles y adultos de ambos sexos. En el cautiverio $\mathrm{C} 2$ se administró a los venados un solo tratamiento antihelmíntico (albendazol, $5 \mathrm{mg} / \mathrm{kg}$ de peso vivo) durante la época de lluvia ( 15 de Julio).

Diez muestras fueron tomadas directamente del suelo y obtenidas cada quince dias durante doce meses, de enero a diciembre de 1995.

Las muestras fueron identificadas por cautiverio y transportadas en refrigeración al laboratorio de Parasitología de la Facultad de Medicina Veterinaria y Zootecnia de la Universidad Autónoma de Yucatán. Fueron procesadas en un periodo no mayor de 24 horas, mediante la técnica cualitativa de Flotación centrifugada y cuantitativa de McMaster (Rodríguez et al. 1994). Las muestras positivas a huevecillos del orden Strongylida fueron transferidas a cultivo de larvas infectantes mediante la técnica de Corticelli-Lai descrita por Rodríguez et al. (1994). Las larvas obtenidas de los cultivos fueron clasificadas de acuerdo a su morfología y tamaño, según el Ministry of Agriculture, Fisheries and Food (1986). Las muestras que contenian ooquistes del orden Coccidia fueron transferidas a cultivos en dicromato de potasio al 2\% para la determinación de géneros.

Durante el periodo de muestreo, se obtuvieron datos climatológicos cada quince dias de la estación metereológica de la Comisión Nacional del Agua, ubicada en la ciudad de Mérida, Yucatán, México. Las variables registradas fueron: temperatura media ambiental (TA), precipitación pluvial (PP) y humedad relativa (HR).

Se contrastaron las frecuencias y cantidad de excresión de ooquistes y huevecillos de los órdenes Coccidia y Strongylida entre cautiverios. Se probó el efecto de dos estaciones anuales: lluvia y seca con respecto a la frecuencia y descarga parasitaria, a través de análisis de 

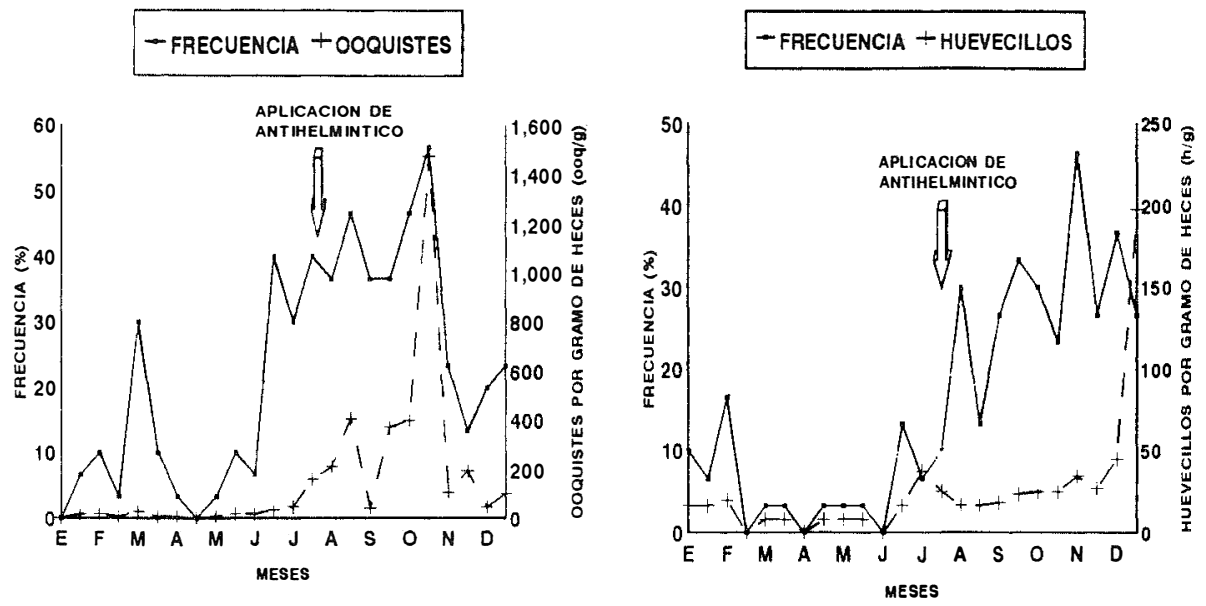

Fig. 1. Frecuencia de parasitosis y excresión de ooquistes del orden Coccidia en venados cola blanca (Odocoileus virginianus yucatanensis) en cautiverio.

Fig. 2. Frecuencia de parasitosis y excresión de huevecillos del orden Strongylida en venados cola blanca (Odocoileus virginianus yucatanensis) en cautiverio.

Kruskal-Wallis, y se correlacionaron los valores de HR, PP y TA con la frecuencia de parasitosis de los órdenes Coccidia y Strongylida, por medio de la prueba de correlación de rangos de Spearman (Downie y Heath 1986).

\section{RESULTADOS}

Se determinaron siete géneros, correspondientes a cinco órdenes de PGI en venado cola blanca, los cuales fueron: Coccidia, cuyos géneros fueron Isospora y Eimeria; Strongylida, representado por los géneros Haemonchus y Cooperia; Rahbditoidea, con un solo género, Strongyloides; Enoplida representado por Trichuris y, finalmente el orden Anoplocephalidea también representado por un solo género, $M o$ niezia. La frecuencia de PGI por órdenes y cautiverio se muestra en el Cuadro 1. Los géneros recuperados y la frecuencia relativa en los coprocultivos que contenian huevecillos del orden Strongylida fueron Haemonchus spp con $98.2 \%$, y Cooperia spp con $1.8 \%$. Los géneros recuperados y la frecuencia relativa en los cultivos que contenian ooquistes del orden Coccidia fueron Eimeria spp con $44 \%$ e Isospora spp con 56\%.

\section{CUADRO 1}

Frecuencia de parasitismo en venados cola blanca (Odocoileus virginianus yucatanensis) en tres cautiverios del Estado de Yucatán, México, en el año de 1995.

\begin{tabular}{lcccc} 
Orden del parásito & \multicolumn{5}{c}{$\begin{array}{c}\text { Frecuencia (\%) en } \\
\text { cada confinamiento }\end{array}$} & Frecuencia (\%) \\
& Cl & C2 & C3 & \\
& & & & \\
Coccidia & 28.4 & 12.4 & 23.2 & 21.3 \\
Strongylida & 21.6 & 5.2 & 19.2 & 15.3 \\
Rhabditoidea & 1.2 & 1.6 & 3.2 & 2.0 \\
Enoplida & 1.6 & 0.4 & 0.0 & 0.66 \\
Anoplocephalidea & 0.4 & 2.8 & 6.4 & 3.2
\end{tabular}

$\mathrm{Cl}$ = Zoológico 1

$\mathrm{C} 2=$ Zoológico 2

$\mathrm{C} 3=$ Criadero semiintensivo

En la Fig. 1 se presentan las frecuencias y la cantidad de excresión de ooquistes del orden Coccidia durante el período de estudio. En la Fig. 2 se presentan las frecuencias y la cantidad de excresión de huevecillos del orden Strongylida durante el mismo período de estudio. 

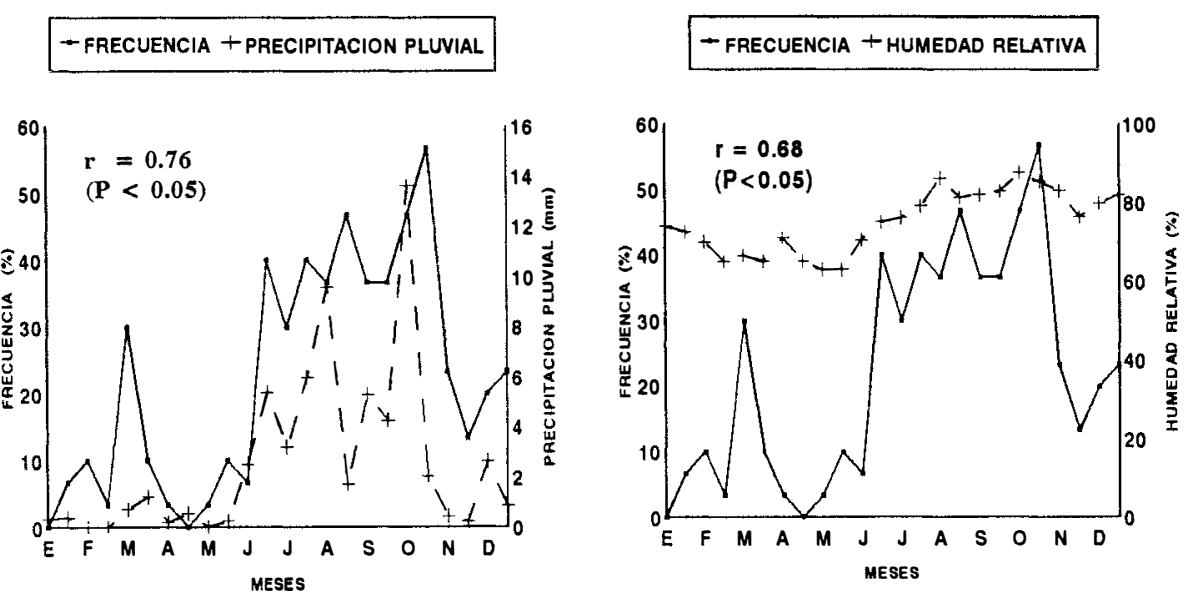

Fig. 3. Variación de la frecuencia de parasitosis por Coccidia en venados cola blanca (Odocoileus virginianus yucatanensis) relacionada con la precipitación pluvial.

Fig. 4. Variación de la frecuencia de parasitosis por Coccidia en venados cola blanca (Odocoileus virginianus yucatanensis) relacionada con la humedad relativa.

Se encontró diferencia significativa en las frecuencias de los ordenes Coccidia y Strongylida entre los tres cautiverios, siendo menor en el C2 (Kruskal-Wallis p<0.05). La cantidad excretada de ooquistes del género Coccidia fue semejante en los tres cautiverios (Kruskal-Wallis $\mathrm{p}>0.05$ ), pero no así para la excresión de huevecillos del orden Strongylida (Kruskal-Wallis $\mathrm{p}<0.05$ ), siendo mayor para el C3.

La frecuencia y excresión de ooquistes del orden Coccidia fue mayor en la época de lluvia (Kruskal-Wallis $\mathrm{p}<0.05$ ) que en la época de seca. La frecuencia y excresión de huevecillos del orden Strongylida fue similar en ambas estaciones del año (Kruskal-Wallis $\mathrm{p}>0.05$ ); sin embargo, se observó una tendencia al incremento en la época de lluvia.

Los PGI de los órdenes Rahbditoidea, Enoplida y Anoplocephalidae se presentaron esporádicamente y sin una tendencia definida durante el año.

Los rangos de variación media quincenal que tuvieron la TA, HR y PP en la época de seca fueron de 17 a $25^{\circ} \mathrm{C}$, de 63 a $82.9 \%$ y de 0.0 a $2.6 \mathrm{~mm}$, respectivamente. En la época de lluvia se registraron variaciones de 23.7 a $25.4^{\circ} \mathrm{C}$ para TA, de 70.4 a $87.4 \%$ para HR y de 1.7 a 13.6 $\mathrm{mm}$ para PP.
Los valores de correlación entre frecuencia de parasitosis por el orden Coccidia con la PP, HR y TA fueron de $0.76,0.68$ y 0.58 , respectivamente (Spearman p<0.05) (Fig. 3, 4 y 5). Las correlaciones significativas encontradas, fueron entre la frecuencia de parasitosis por el orden Strongylida con PP y HR, cuyos valores fueron de 0.46 y 0.83 , respectivamente (Spearman p<0.05) (Fig. 6 y 7).

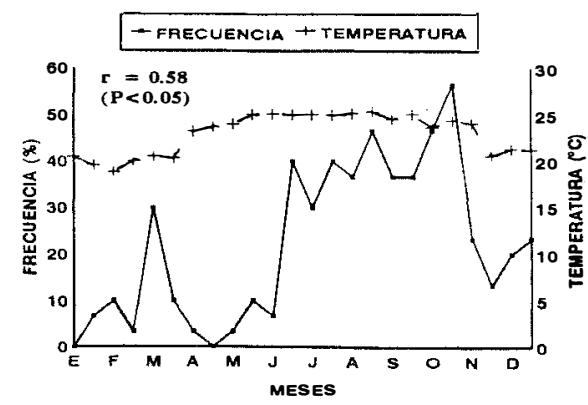

Fig. 5. Variación de la frecuencia de parasitosis por Coccidia en venados cola blanca (Odocoileus virginianus yucatanensis) relacionada con la temperatura ambiental. 

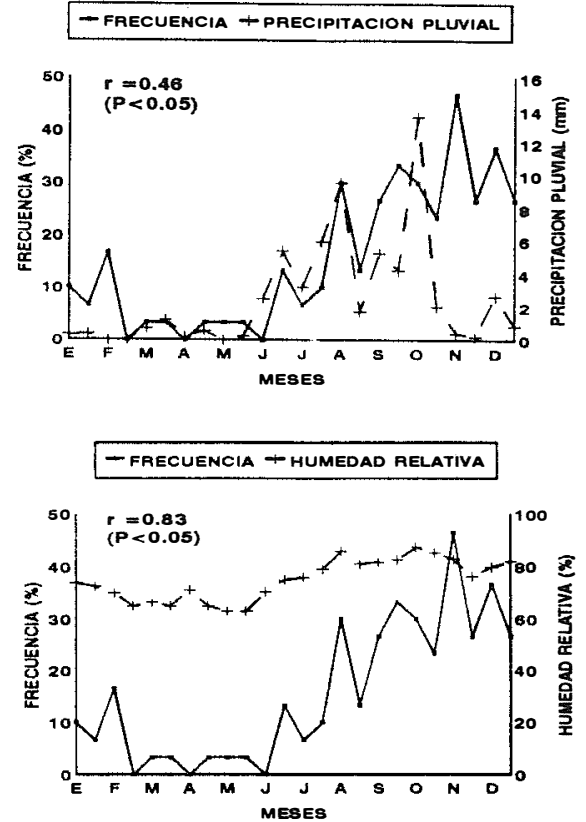

Fig. 6. Variación de la frecuencia de parasitosis por Strongylida en venados cola blanca (Odocoileus virginianus yucatanensis) relacionada con la precipitación pluvial.

Fig. 7. Variación de la frecuencia de parasitosis por Strongylida en venados cola blanca (Odocoileus virginianus yucatanensis) relacionada con la humedad relativa.

\section{DISCUSION}

De los siete géneros de PGI reportados en el presente estudio, seis han sido previamente determinados en venados y rumiantes domésticos que habitan lugares de clima templado y tropical (Soulsby 1987, Domínguez et al. 1988, Jaimes et al. 1993; Torres et al. 1995); sin embargo, se reporta por primera vez la presencia del género Isospora como PGI de venado cola blanca, en Yucatán, México. El género Isospora parasita comunmente a cerdos no a rumiantes (Blood \& Radostits .1989); por el momento no es posible explicar con certeza el origen de la parasitosis por Isospora en venados, puesto que no existe algún intercambio de material fecal o alimenticio entre los criaderos de venado estudiados con granjas porcinas; sin embargo, en los tres criaderos se encontró alta frecuencia de Isospora.
Los géneros Haemonchus, Isospora y Eimeria fueron los PGI más frecuentes en venados cola blanca de la región central del estado de Yucatán, México. Se ha reportado que en venados y en rumiantes domésticos, los géneros Haemonchus y Eimeria ocasionan retraso en el crecimiento, debilidad, anemia, disminución de la resistencia a otros padecimientos y reducción del apetito (Prestwood et al. 1973, Abbas et al. 1987, Domínguez et al. 1993). La principal característica de la infección por el género Haemonchus es la anemia; causada tanto por efecto de las larvas de cuarto estadio como por los adultos, que son hematófagos y se calcula que en un animal parasitado, la pérdida media de sangre es de $0.05 \mathrm{ml}$ al día por parásito (Soulsby 1987). La patogenicidad del orden Coccidia se debe a su capacidad de reproducción y destrucción de gran cantidad de células intestinales (Soulsby 1987, Quiróz 1989).

La frecuencia y dinámica de excresión de huevecillos del orden Strongylida fue menor en el C2, esto fue probablemente debido a la alta efectividad del antihelmíntico (albendazol) aplicado a los venados en la época de lluvia. Theodorides et al. (1976) mencionan que el albendazol es un excelente antihelmíntico y tiene una eficacia hasta del $97 \%$ contra nemátodos del género Haemonchus.

Un hecho importante fue el aumento de la frecuencia y excresión de ooquistes del orden Coccidia en la época de lluvia. Para que un ooquiste sea infectante es necesario que se realice la esporulación en el ambiente externo, para ello se requiere de tres elementos principales: oxígeno, temperatura de 20 a $24^{\circ} \mathrm{C}$ y humedad ambiental superior al 70\% (Soulsby 1987, Quiróz 1989). En la zona centro del estado de Yucatán, México, la PP, TA y HR registradas en la estación de lluvia fueron adecuadas para el desarrollo del orden Coccidia en el medio externo, lo que permitió mayor riesgo de infección en los venados. En la época de seca se presentaron meses con HR inferiores al 70\%, lo que provocaron la desecación de los ooquistes en el ambiente y la baja infección de los venados. Esta tendencia en la PGI, ha sido reportado también en bovinos de clima tropical (Rodríguez et al. 1988, Ali y Latif 1989). 
De igual manera, el orden Strongylida presentó una correlación positiva con la HR; este hallazgo es el resultado del desarrollo de huevecillos y el movimiento de larvas infectantes, principalmente del género Haemonchus; las cuales depende de una HR entre 70 y $100 \%$, lo cual ocurre en la época de lluvia (Delgado 1989).

La cantidad de ooquistes del orden Coccidia (200-400 ooquistes por gramo de heces) y huevecillos del orden Strongylida (50-200 huevecillos por gramo de heces) excretados durante la época de máximo riesgo, ha sido usado en otros rumiantes para evaluar el grado de parasitismo (Rodríguez et al. 1994); sin embargo, en venados cola blanca no hay suficiente información para hacer un análisis al respecto. Se necesitan realizar estudios más precisos para determinar si la frecuencia y/o las cargas parasitarias reportadas en el presente estudio tienen un efecto significativo sobre el comportamiento productivo o reproductivo del venado cola blanca mantenidos en cautiverio en esta región de México.

\section{RESUMEN}

El objetivo del presente estudio fue determinar los géneros y estimar la cantidad de huevecillos y ooquistes de parásitos gastrointestinales (PGI) excretados durante un año en heces de venados cola blanca (Odocoileus virginianus yucatenensis) mantenidos en cautiverio en Yucatán, México. Se recolectaron muestras fecales en tres confinamientos ubicados en la zona centro del estado de Yucatán, de enero a diciembre de 1995. Diez muestras fueron colectadas de cada confinamiento cada dos semanas. Las muestras fueron procesadas mediante la técnica de Flotación Centrifugada y McMaster. Se realizaron cultivos de heces para obtener larvas infectantes $\mathrm{L}_{3}$ mediante la técnica de Corticelli-Lai. Las heces positivas a ooquistes fueron cultivados en dicromato de potasio al 2\%. Se determinaron siete géneros (Haemonchus spp, Cooperia spp, Isospora spp, Eimeria spp, Trichuris spp, Strongyloides spp y Moniezia spp) correspondientes a cinco órdenes. Haemonchus spp, Isospora spp y Eimeria spp fueron los géneros más frecuentes. El género Isospora se reporta por primera vez en venados de la región, aunque no fue posible explicar el origen de este hallazgo. La frecuencia y nivel de excresión de huevecillos y ooquistes de PGI fue variable durante el año, con incremento en la época de lluvia. Se encontró correlación positiva entre humedad relativa, temperatura ambiente y precipitación pluvial con los órdenes Coccidia y Strongylida. En la zona centro del estado de Yucatán, México, las condiciones meteorológicas en la época de lluvia son propicias para el desarrollo del parasitismo gastrointestinal, lo que permite mayor riesgo de infección en los venados.

\section{AGRADECIMIENTOS}

El presente trabajo de investigación fue financiado por FUNDACION FORD-PROTROPICO, convenio No. 920604. Los autores agradecen a las autoridades del Parque Zoológico del Centenario, Zoológico de Cholul y Criadero de venados de Cementos Maya, las facilidades para la realización de este trabajo.

\section{REFERENCIAS}

Abbas, B., G. Post \& W.C. Marquardt. 1987. Merogony and gametogony of Eimeria mccordocki (Protozoa Eimeridae) in the mule deer, Odocoileus h. hemionus. Vet. Parasitol. 24:1-5.

Ali, S.R. \& B.M. Latif. 1989. Bovine coccidiosis in Baghdad area Iraq. J. Biol. Sci. 20:483-488.

Blood, D.C. \& O.M. Radostits. 1989. Veterinary Medicine: A textbook of the diseases of cattle, sheep, pigs, goats and horses. Bailliere Tindall. Oxford, Gran Bretaña. $1502 \mathrm{p}$.

Anónimo. 1970. Centro de Investigaciones Agrícolas de la Península de Yucatán (CIAPY). Cultivos importantes para la Diversificación Agrícola en la Península de Yucatán. Instituto Nacional de Investigaciones Agrícolas. Secretaría de Agricultura y Ganadería. Centro de Investigaciones Agrícolas de la Península de Yucatán 4:1-38.

Carrillo, M. F. 1987. Políticas sobre la administración del venado cola blanca en cautiverio. Rev. Univ. Autón. 162:78-85.

Delgado, A. 1989. Comportamiento de las larvas de estrongilatos del bovino en el ambiente externo y su importancia en el control de estas helmintosis. Rev. Cuba. Cien. Vet. 20:127-142.

Domínguez, J., F. Victoria \& J. Ramírez. 1988. Estudio preliminar de las parasitosis gastrointestinales que afectan al venado cola blanca (Odocoileus virginianus) criado en las condiciones de Yucatán. Resumen del VIII Congreso Nacional de Parasitología. Universidad 
Autónoma de Hidalgo. Escuela de Medicina. C.E.U.N.I. 11-13 de Octubre. Pachuca, Hidalgo. México. 179 p.

Domínguez, A.J.L., V.R.I. Rodríguez \& N. Honhold. 1993. Epizootiología de los parásitos gastrointestinales en bovinos del estado de Yucatán. Vet. Méx. 24:189-193.

Downie, N.M. \& R.W. Heath. 1986. Métodos Estadísticos Aplicados. Harla. México, D.F. 380 p.

Dunn, A. 1983. Helmintología veterinaria. El Manual Moderno. México, D.F. 390 p.

Flores, A.F.J. 1990. Ganadería diversificada: Futuro sostenible para la conservación de la fauna silvestre. VIII Simposio sobre fauna silvestre. Facultad de Medicina Veterinaria y Zootecnia, Universidad Nacional Autónoma de México. México, D.F. 403 p.

García, E. 1988. Modificaciones al sistema de clasificación climática de Köppen. Cuarta edición. Instituto de Geografía. Universidad Nacional Autónoma de México. México, D.F. 211 p.

Jaimes, B., A. Orozco y V. Rodríguez. 1993. Análisis de excretas del venado cola blanca (Odocoileus virginianus) para la detección de nemátodos endoparásitos gastrointestinales del Parque El Ocotal. XI Simposio Nacional y I Simposio Internacional de Fauna Silvestre. Facultad de Medicina Veterinaria y Zootecnia, Universidad Nacional Autónoma de México, UMKA y Gobierno del Estado de Tabasco. Tabasco, México. 231 p.

Ministry of Agriculture Fisheries and Food. 1986. Manual of Veterinary Parasitological Laboratory Techniques. Reference Book 418. Her Majesty's Stationary Office, Londres. $159 \mathrm{p}$.

Olvera, G.A.A. 1991. Manual de manejo del venado cola blanca (Odocoileus virginianus) en cautiverio. Tesis de licenciatura, Facultad de Estudios Superiores Cuautitlán. Universidad Nacional Autónoma de México. Cuautitlán, Estado de México. México.

Prestwood, A., F. Hayes, J. Hammand-Eve \& J. Smith. 1973. Abomasal helminths of white-tailed deer in Southeastern United State, Texas, and the Virgin Islands. J.A.V.M.A. 163:556-561.

Quinto, F. 1994. Avances para el manejo de venados en selvas tropicales del sureste de México. IV Simposio sobre venados de México. Nuevo Laredo, Tamaulipas. México. 245 p.
Quiróz, H. 1989. Parasitología y enfermedades parasitarias en animales domésticos. Limusa. México, D.F. 876 p.

Rivera, M.M. 1991. Identificación y cuantificación de helmintos gastrointestinales del venado cola blanca (Odocoileus virginianus) en un rancho cinegético del estado de Nuevo León. Tesis de licenciatura, Universidad Nacional Autónoma de México, México, D.F. México.

Roa, R. M. 1986. El venado cola blanca como animal de zoológico. I Simposio sobre el venado en México. Facultad de Medicina Veterinaria y Zootecnia, Universidad $\mathrm{Na}$ ional Autónoma de México, México D.F. México. 201 p.

Rodríguez, N., M. Percebo \& E. Fuentes. 1988. Coccidiosis de invierno con presencia de Eimeria zuerni. Rev. Cuba. Cienc. Vet. 19:93-98.

Rodríguez, V.R.I., A.J.L. Domínguez \& G.L.A. Cob. 1994. Técnicas diagnósticas de parasitología veterinaria. Universidad Autónoma de Yucatán. Mérida, Yucatán, México. 236 p.

Rogel, B.A.C. \& A.S.L. Luján. 1982. Análisis preliminar sobre el ciclo de vida del venado cola blanca (Odocoileus virginianus yucatanensis) en cautividad, Tekax, Yucatán. Reporte Anual. Dirección General de Fauna Silvestre. Subprograma de Fauna Silvestre. Secretaría de Agricultura y Recursos Hidráulicos. Yucatán, México. $18 \mathrm{p}$.

Soulsby, E.J.L. 1987. Parasitología y enfermedades parasitarias en los animales domésticos. Nueva Interamericana, México, D.F. México. 823 p.

Torres-Acosta, J.F., R.I. Rodríguez-Vivas \& R. CámaraSarmiento. 1995. Efecto del parto sobre la eliminación de huevecillos de nemátodos y ooquistes de Eimeria gastrointestinales en cabras criollas. Rev. Biomed. 6:208-215.

Theodorides, V.J., R.J. Gyurik, \& M.D. Kingsbury. 1976. Anthelmintic activity of albendazole against liver flukes, tapeworms lung and gastrointestinal roundworms. Experientia 32:702-703.

Woolf, A. \& J. Harder. 1979. Population dynamics of a captive white-tailed deer herd with emphasis on reproduction and mortality. Wildl. Monogr. 67:5-53. 